\title{
Information Flow, Non-Markovianity and Geometric Phases
}

\author{
S. L. Wu, ${ }^{1}$ X. L. Huang, ${ }^{1,2}$ L. C. Wang, ${ }^{1}$ and X. X. $\mathrm{Yi}^{1, \text {, }}$ \\ ${ }^{1}$ School of Physics and Optoelectronic Technology, \\ Dalian University of Technology, Dalian 116024 China \\ ${ }^{2}$ School of Physics and Electronic Technology, \\ Liaoning Normal University, Dalian, 116029, China
}

\begin{abstract}
Geometric phases and information flows of a two-level system coupled to its environment are calculated and analyzed. The information flow is defined as a cumulant of changes in trace distance between two quantum states, which is similar to the measure for non-Markovianity given by Breuer. We obtain an analytic relation between the geometric phase and the information flow for pure initial states, and a numerical result for mixed initial states. The geometric phase behaves differently depending on whether there are information flows back to the two-level system from its environment.
\end{abstract}

PACS numbers: 03.65.Yz, 03.65.Vf, 03.65.Ta

\section{INTRODUCTION}

Geometric phase has not been noticed for a long time until Pancharatnam's study [1] and Berry's discovery [2]. Shortly, Simon gave a geometric interpretation of this kind of phase in the language of differential geometry and fibre bundles [3]. Since then there was a keen interest in holonomy effect in quantum theory, which leads to many extensions of the geometric phase, including the geometric phase acquired in a non-adiabatic and cyclic evolution by Aharonov and Anandan [4], in a non-adiabatic and non-cyclic evolution by Samel and Bhandari [5] and in a non-adiabatic, non-cyclic and non-unitary evolution by Mukunda [6]. All those investigations were focused on pure state. For a practical quantum system, however, its state would be mixed due to the unavoidable coupling to its environment. This motivates the study on the geometric phase for mixed states [7 [10], which was defined by [10],

$$
\Phi_{\mathrm{GP}}(t)=\operatorname{Arg}\left(\sum_{i=1}^{N} \sqrt{\epsilon_{i}(0) \epsilon_{i}(t)}\left\langle\psi_{i}(0) \mid \psi_{i}(t)\right\rangle \exp \left\{-\int_{0}^{t}\left\langle\psi_{i}\left(t^{\prime}\right) \mid \dot{\psi}_{i}\left(t^{\prime}\right)\right\rangle d t^{\prime}\right\}\right),
$$

where $\epsilon_{i}(t)$ and $\left|\psi_{i}(t)\right\rangle$ are the eigenvalues and the corresponding eigenstates of the density matrix, respectively. Throughout this paper, we will use this definition to study the geometric phase.

On the other hand, it is complicated to exactly describe the dynamics of open systems due to the huge number of variables in an environment. In order to simplify the description, one could make some approximations such as the weakly coupling and the Markovian approximation. Under these approximations, we can obtain a Markovian master equation that describes the dynamics of the open system without memory of its history. However, many systems exhibit strong non-Markovian effect and can not be described by the Markovian master equation. It is then interesting to study the geometric phase in a non-Markovian dynamics, and establish the relation between the geometric phase and the non-Markovianity of the dynamics.

The non-Markovianity may be defined in many ways [14 17], for instance, in Ref. [14] the authors proposed a scheme to quantify the degree of the non-Markovianity based on the trace distance of two quantum states 14], and in Ref. 15] the non-Markovianity was measured by exploiting the specific traits of quantum correlations. The effects of nonMarkovianity on geometric phase has been considered by several works, e.g. Refs. [12] and [13]. In this paper, we will establish a relation between the geometric phase and the information flows. We divide the information flow into two types, i.e., the information flow from the open system into the environment $\mathcal{M}$ (call forward information flow) and the information flow back from the environment to the open system $\mathcal{N}$ (call backward information flow). The backward information flow $\mathcal{N}$ in fact is a modified measure of non-Markovianity given by Breuer and his co-workers. This paper is organized as follows. In Sec.II, we present a definition for the information flow based on the trace distance, then we establish the relation between the information flow and the geometric phase for pure initial states. The case of mixed initial states is considered in Sec.III, where the geometric phase and the information flow are calculated numerically. Finally, we present conclusion and discussions in Sec.IV.

*Electronic address: yixx@dlut.edu.cn 


\section{INFORMATION FLOW AND GEOMETRIC PHASE FOR PURE INITIAL STATES}

\section{A. A measure for information flow}

Here we first recall the measure for non-Markovianity defined by Breuer [14]. This definition is based on the so-called trace distance between two states $\rho_{1}$ and $\rho_{2}$

$$
D\left(\rho_{1}, \rho_{2}\right)=\frac{1}{2} \operatorname{Tr}\left\|\rho_{1}-\rho_{2}\right\|,
$$

where $\|A\|=\sqrt{A A^{\dagger}}$. For a two-level system, this trace distance is equal to one half of the ordinary Euclidean distance between the two states on the Bloch sphere, i.e. $D\left(\rho_{1}, \rho_{2}\right)=\frac{1}{2}\left|\vec{r}_{1}-\vec{r}_{2}\right|$, where $\vec{r}_{j}$ is the Bloch vector for state $\rho_{j}$. The change rate of the trace distance can be represented as

$$
\sigma\left(\rho_{1}(t), \rho_{2}(t)\right)=\frac{d}{d t} D\left(\rho_{1}(t), \rho_{2}(t)\right)
$$

When $\sigma<0, \rho_{1}(t)$ and $\rho_{2}(t)$ approach to each other in the dynamics and this can be understood as information flow from the system to the environment; when $\sigma>0, \rho_{1}(t)$ and $\rho_{2}(t)$ is away from each other in the time evolution, and this can be interpreted as information flow back to the system, which is treated as a typical character of the non-Markovianity. As shown in Refs. [14] and [18], one can define a measure of non-Markovianity $\mathcal{N}_{B}(t)$ for a quantum process $\Psi(t)$ by maximizing over all initial states $\left(\rho_{1}(0), \rho_{2}(0)\right)$ of total gain of the trace distance, namely,

$$
\mathcal{N}_{B}(\Psi)=\max _{\rho_{1,2}(0)} \int_{\sigma>0} \sigma\left(\rho_{1}(t), \rho_{2}(t)\right) d t .
$$

The measure of non-Markovianity only characterizes the gain of the trace distance in the dynamics. To describe the loss and gain of the trance distance uniformly, we extend the concept of information flow in the following. In fact, one of the information flows defined below is a modified measure of non-Markovianity in Eq.(4). We choose a steady state as $\rho_{2}$ in Eq. (2) and call it standard state. This choice makes the maximization easy, however, it can not measure all non-Markovian dynamics. Fortunately, this simplified measure of non-Markovianity is in agreement with the measure given in [14] in our case.

Now we define $\mathcal{M}$ and $\mathcal{N}$ to measure the information gain and lose for a given initial state $\rho_{1}(0)$ in the dynamics $\Psi(t)$. We will refer this information gain and loss uniformly as formation flows.

$$
\mathcal{N}(\Psi)=\int_{\sigma>0} \sigma\left(\rho_{1}(t), \rho_{2}\right) d t
$$

and

$$
\mathcal{M}(\Psi)=-\int_{\sigma<0} \sigma\left(\rho_{1}(t), \rho_{2}\right) d t .
$$

Obviously, the above two measures satisfy,

$$
D\left(\rho_{1}(t), \rho_{2}\right)=D\left(\rho_{1}(0), \rho_{2}\right)+\mathcal{N}\left(\rho_{1}(t), \rho_{2}\right)-\mathcal{M}\left(\rho_{1}(t), \rho_{2}\right) .
$$

The difference between $\mathcal{N}(t)$ and $\mathcal{N}_{B}$ is as follows. $\mathcal{N}_{B}$ is the maximum information flow back to the system in the dynamics. Hence it does not depend on the initial condition. However $\mathcal{N}(t)$ is the information flow back to system with respect to the standard state $\rho_{2}(0)$ in the interval $(0, t)$ (assumed that a steady state for quantum process $\Psi(t)$ exists, and the standard state $\rho_{2}(0)$ is exactly the steady state as before). This simplification is true in the situation considered in this paper, i.e., a two-level system coupling to vacuum electromagnetic fields at zero temperature with standard state $\rho_{2}$ in the Bloch sphere representation as $\vec{r}_{2}=(0,0,-1)$.

With these definition and notations, we will discuss the relationship between the geometric phase and the information flows between the open system and its environment.

\section{B. The geometric phase for pure initial states}

Consider a two-level system coupled to its environment at zero temperature. The general form of the density matrix can be expressed as $\rho(t)=\frac{1}{2}(1+\vec{r}(t) \cdot \vec{\sigma})$, where $\vec{\sigma}=\left(\sigma_{x}, \sigma_{y}, \sigma_{z}\right)$ is the Pauli matrices, and $\vec{r}(t)=r(t)$. 
$(\sin \theta(t) \cos \phi(t), \sin \theta(t) \sin \phi(t), \cos \theta(t))$ is the Bloch vector. For pure initial states $|\vec{r}(0)|^{2}=1$, while for mixed initial states $|\vec{r}(0)|^{2}<1$. It is easy to obtain the instantaneous eigenvalues of the above density matrix $\rho(t)$ as,

$$
\epsilon_{ \pm}(t)=\frac{1}{2}(1 \pm|\vec{r}(t)|)
$$

It is obvious that for the pure initial states, the eigenvalue $\epsilon_{-}(t=0)=0$, which means that the eigenstate corresponding to the eigenvalue $\epsilon_{-}(t)$ gives no contribution to the geometric phase. This simplifies our calculation and helps us to obtain an analytic result for the geometric phase. The eigenstates corresponding to the eigenvalues in Eq.(8) can be written as,

$$
\left|\psi_{+}(t)\right\rangle=\left(\begin{array}{c}
\sin \frac{\theta(t)}{2} \\
\cos \frac{\theta(t)}{2} e^{i \phi(t)}
\end{array}\right),\left|\psi_{-}(t)\right\rangle=\left(\begin{array}{c}
-\cos \frac{\theta(t)}{2} \\
\sin \frac{\theta(t)}{2} e^{i \phi(t)}
\end{array}\right) .
$$

Now substitute Eq.(8) and Eq.(9) into the Eq.(1) with an assumption that $\phi=\omega_{0} t+\phi_{0}$ (where $\omega_{0}$ and $\phi_{0}$ are constants, this is reasonable for different kinds of master equation [12, 13]), the geometric phase with pure initial states (their Bloch vector is $\vec{r}(0)$ ) can be obtained as

$$
\Phi_{\mathrm{GP}}=-\int_{0}^{T} \omega_{0} \cos ^{2} \frac{\theta(t)}{2} d t
$$

where we set $T=\frac{2 n \pi}{\omega_{0}}(n=1,2,3, \ldots)$, i.e., a multiple of the quasi-period $\frac{2 \pi}{\omega_{0}}$.

Next, we establish the relationship between the geometric phase and the information flows $\mathcal{M}(t)$ and $\mathcal{N}(t)$. Keeping the relationship Eq.(17) between the $\mathcal{N}(t)$ and $\mathcal{M}(t)$ in mind, we obtain the geometric phase acquired by the system,

$$
\Phi_{\mathrm{GP}}=-\int_{0}^{T} \omega_{0}\left[\frac{1}{2}+\frac{r_{z}(t)}{2 \sqrt{4[D(0)+\mathcal{N}(t)-\mathcal{M}(t)]^{2}-2 r_{z}(t)-1}}\right] d t
$$

where $r_{z}(t)=r(t) \cos \theta(t)$ is the z-component of $\vec{r}(t)$ and $D(0)=D\left(\rho_{1}(0), \rho_{2}\right)$. It is shown that when the system is closed, i.e. $\cos \theta(t)=\cos \theta_{0}$ and $r(t)=1$, where $\theta_{0}$ is initial polar angle on the Bloch sphere, and setting $T=2 \pi / \omega_{0}$, Eq.(11) reduced to the well-known form, $\Phi_{\mathrm{GP}}^{(\text {closed })}=-\pi\left(1+\cos \theta_{0}\right)$, that is the geometric phase acquired by a two-level quantum system in a rotating magnetic field. For a Markovian process, $\mathcal{N}(t)$ is always zero and $\mathcal{M}(t)$ increases with time until it approaches $D(0)$. In this case, the geometric phase is only influenced by the information flow to the environment. When we consider the non-Markovian effects, the situation is more complicated, and the information flow $\mathcal{N}(t)$ back to the open system has a link to the geometric phase given by Eq.(11). We will discuss it numerically in Sec.III.

\section{THE GEOMETRIC PHASE FOR MIXED STATE}

In this section, we will study the geometric phase of a two-level system with mixed initial states. Because it is difficult to get an analytical result like Eq.(11) for the information flows and the geometric phase, we here numerically and perturbatively establish a relation between the geometric phase and the information flow. The perturbation is carried out to the first order in the coupling constant, while the numerical results are for a wide range of coupling constants. Two types of master equation, the time-local master equation and the memory kernel master equation with exponential memory, will be considered.

\section{A. The time-local master equation}

Let us consider a two-level system interacting with a vacuum field at zero temperature whose spectral density is Lorentzian [11, 19, 20],

$$
J(\omega)=\frac{1}{\pi} \frac{W^{2} \lambda}{\left(\omega_{0}-\omega\right)^{2}+\lambda^{2}} .
$$

Here $W$ is the coupling constant between the system and the environment, $\omega_{0}$ is the atomic transition frequency which is of the time scale $\tau_{0} \sim \omega_{0}^{-1}$, and $\lambda$ is the spectral width of the coupling that is connected to the environment 
correlation time, $\tau_{B} \sim \lambda^{-1}$. The dynamics of this system is governed by the following master equation (namely the time-local master equation) [11],

$$
\dot{\rho}(t)=-i \Delta(t)\left[\sigma_{+} \sigma_{-}, \rho(t)\right]+\Gamma(t)\left[2 \sigma_{-} \rho(t) \sigma_{+}-\sigma_{+} \sigma_{-} \rho(t)-\rho(t) \sigma_{+} \sigma_{-}\right],
$$

where $\sigma_{ \pm}$are the pauli operators and the parameters

$$
\Delta(t)=-\Im\left[\frac{\dot{c}(t)}{c(t)}\right], \Gamma(t)=-\Re\left[\frac{\dot{c}(t)}{c(t)}\right]
$$

play the role of Lamb shift and decay rate for the system, respectively. Here $c(t)$ can be calculated by means of Laplace transform as,

$$
c(t)=\exp \left[\frac{-\left(\lambda+i \omega_{0}\right) t}{2}\right]\left(\cosh \frac{\Omega t}{2}+\frac{\lambda}{\Omega} \sinh \frac{\Omega t}{2}\right)
$$

with $\Omega=\sqrt{\lambda^{2}-4 W^{2}}$. We note that the rate $R=\frac{W}{\lambda}$ indicates the strength of the non-Markovianity. With $R<\frac{1}{2}$, the dynamics is called time-dependent Markovian, while for $R>\frac{1}{2}$ the dynamics is non-Markovian. Here we assume again that the initial state of the open system is

$$
\rho(0)=\frac{1}{2}\left(1+\vec{r}_{0} \cdot \vec{\sigma}\right)
$$

where $\vec{r}_{0}=r_{0} \cdot\left(\sin \theta_{0} \cos \phi_{0}, \sin \theta_{0} \sin \phi_{0}, \cos \theta_{0}\right)$ and $\left|\vec{r}_{0}\right|^{2}<1$. With this initial condition, the density matrix of the system at time $t$ can be obtained from the master equation Eq.(13)

$$
\rho(t)=\frac{1}{2}\left(\begin{array}{cc}
\left(1+r_{0} \cos \theta_{0}\right)|c(t)|^{2} & r_{0} \sin \theta_{0} \exp \left(i \phi_{0}\right) c(t) \\
r_{0} \sin \theta_{0} \exp \left(-i \phi_{0}\right) c^{*}(t) & 2-\left(1+r_{0} \cos \theta_{0}\right)|c(t)|^{2}
\end{array}\right),
$$

The eigenvalues and the eigenstates of the reduced density matrix Eq. (17) can be easily obtained as

$$
\begin{aligned}
\epsilon_{ \pm}(t) & =\frac{1}{2}(1 \pm r(t)) \\
\left|\psi_{+}(t)\right\rangle & =\left(\begin{array}{c}
\sin \frac{\theta_{t}}{2} \\
\cos \frac{\theta_{t}}{2} e^{i\left(\omega_{0} t+\phi_{0}\right)}
\end{array}\right) \\
\left|\psi_{-}(t)\right\rangle & =\left(\begin{array}{c}
-\cos \frac{\theta_{t}}{2} \\
\sin \frac{\theta_{t}}{2} e^{i\left(\omega_{0} t+\phi_{0}\right)}
\end{array}\right)
\end{aligned}
$$

where $\tan \theta_{t}=\frac{r_{0} \sin \theta_{0}|c(t)|}{\left(1+r_{0} \cos \theta_{0}\right)|c(t)|^{2}-1}$ and $r(t)=\sqrt{\left[\left(1+r_{0} \cos \theta_{0}\right)|c(t)|^{2}-1\right]^{2}+r_{0}^{2} \sin ^{2} \theta_{0}|c(t)|^{2}}$.

We can expand the geometric phase with respect to the coupling strength $W^{2}$ up to the first order (i.e., in the weak coupling limit), that is

$$
\Phi_{\mathrm{GP}}(T) \doteq \Phi_{\mathrm{GP}}^{(0)}-W^{2}\left[\tan \Phi_{\mathrm{GP}}^{(0)} C_{1}\left(r_{0}, \theta_{0}\right) \kappa_{1}(\lambda, T)+\omega_{0} \cos ^{-2} \Phi_{\mathrm{GP}}^{(0)} C_{2}\left(r_{0}, \theta_{0}\right) \kappa_{2}(\lambda, T)\right],
$$

where $\kappa_{1}(\lambda, T)=\left.\frac{\partial|c(T)|^{2}}{\partial W^{2}}\right|_{W^{2}=0}=\frac{1-\exp (-\lambda T)}{\lambda^{2}}-\frac{T}{\lambda}, \kappa_{2}(\lambda, T)=\left.\int_{0}^{T} \frac{\partial|c(t)|^{2}}{\partial W^{2}}\right|_{W^{2}=0} d t=\frac{T}{\lambda^{2}}+\frac{1}{\lambda^{3}}[\exp (-\lambda T)-1]-$ $\frac{T^{2}}{2 \lambda}$, and $\Phi_{\mathrm{GP}}^{(0)}$ is the geometric phase acquired under the unitary evolution with mixed initial states, $\Phi_{\mathrm{GP}}^{(0)}=$ $\arctan \left(r_{0} \tan \left(-i \pi\left(1+\cos \theta_{0}\right)\right)\right)$. The parameter $C_{i}\left(r_{0}, \theta_{0}\right)(i=1,2)$ is a constant relative to the initial condition given by

$$
\begin{gathered}
C_{1}\left(r_{0}, \theta_{0}\right)=\frac{1}{4}\left(r_{0}+r_{0} \cos ^{2} \theta_{0}+2 \cos \theta_{0}\right), \\
C_{2}\left(r_{0}, \theta_{0}\right)=\frac{1}{r_{0}}\left(1+\frac{r_{0} \sin ^{2} \theta_{0} \cos \theta_{0}}{2}-\cos ^{2} \theta_{0}\right) .
\end{gathered}
$$

It is interesting to calculate the trace distance between $\rho(t)$ defined in Eq.(17) and the standard state in the weak coupling limit. Substituting Eq.(17) into Eq.(2), and expanding the trace distance up to the first order in $W^{2}$, we have

$$
\left.D(t) \doteq D(t)\right|_{W^{2}=0}+\frac{W^{2}}{\left.4 D(t)\right|_{W^{2}=0}} \times\left.\left[r_{0}^{2}\left(1+\cos ^{2} \theta_{0}\right)+2 r_{0} \cos \theta_{0}\right] \frac{\partial|c(t)|^{2}}{\partial W^{2}}\right|_{W^{2}=0}
$$




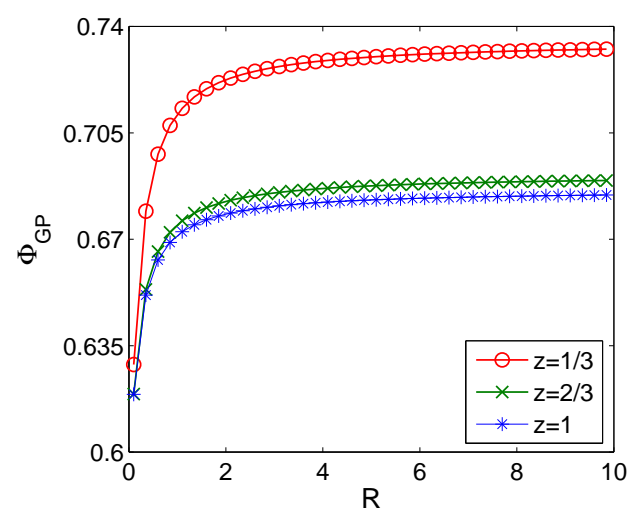

FIG. 1: (Color online) The geometric phase (in units of $\pi$ ) as a function of the parameter $R$ with different $z$. The coupling strength is $W=0.1 \omega_{0}$ and $\omega_{0}=1$. The azimuthal angle of the two components of the initial state are chosen as $\vartheta_{0}=\pi / 4$, $\varphi_{0}=\frac{\pi}{3}$.

where $\left.D(t)\right|_{W^{2}=0}=\sqrt{r_{0}^{2}+1+2 r_{0} \cos \theta_{0}} / 2$ and $\left.\frac{\partial|c(t)|^{2}}{\partial W^{2}}\right|_{W^{2}=0}=\frac{1-\exp (-\lambda t)}{\lambda^{2}}-\frac{t}{\lambda} .\left.\quad D(t)\right|_{W^{2}=0}$ is the trace distance at time $t$ under unitary evolution, it is not difficult to prove that $\left.D(t)\right|_{W^{2}=0}=D(0)$. Then according to Eq.(7), we have

$$
\mathcal{N}(t)-\mathcal{M}(t) \doteq \frac{W^{2}}{\left.4 D(t)\right|_{W^{2}=0}} \times\left.\left[r_{0}^{2}\left(1+\cos ^{2} \theta_{0}\right)+2 r_{0} \cos \theta_{0}\right] \frac{\partial|c(t)|^{2}}{\partial W^{2}}\right|_{W^{2}=0} .
$$

If coupling strength is very weak, there is no information flow back into the system in a quasi-period, i.e. $\mathcal{N}(T)=0$. So it is straightforward to obtain,

$$
\mathcal{M}(t) \doteq-\frac{W^{2}}{\left.4 D(t)\right|_{W^{2}=0}} \times\left.\left[r_{0}^{2}\left(1+\cos ^{2} \theta_{0}\right)+2 r_{0} \cos \theta_{0}\right] \frac{\partial|c(t)|^{2}}{\partial W^{2}}\right|_{W^{2}=0} .
$$

This result tells us that $\mathcal{M}(t)$ increases monotonically with the increasing of $1 / \lambda$. Comparing this result with Eq.(19), one may find that the dependence of $\mathcal{M}(T)$ and $\Phi_{\mathrm{GP}}(T)$ on the spectral width $\lambda$ is almost the same for mixed initial states in the weak coupling limit.

Numerical results for the geometric phase and the information flow $\mathcal{M}$ under the weak coupling limit is shown in Fig 1 and Fig 2, The initial states are chosen as ,

$$
\rho(0)=\frac{1-z}{2} \mathbf{I}+z|\xi\rangle\langle\xi|,
$$

where $|\xi\rangle=\cos \vartheta_{0}|0\rangle+\sin \vartheta_{0} \exp \left(i \varphi_{0}\right)|1\rangle$ is a pure state, $z \in[0,1]$ and $\mathbf{I}$ is a $4 \times 4$ unitary matrix. For $z=0$, the density matrix is the maximumlly mixed state, while they reduce to a pure one in the case of $z=1$. In the language of Bloch vector, the initial state Eq.(24) can be represented as

$$
r_{0}=z, \theta_{0}=2 \vartheta_{0}, \phi_{0}=\varphi_{0} .
$$

Now we establish the relation between the geometric phase and the information flow with different coupling strengths one by one. E.g., $W=0.1 \omega_{0}, W=\omega_{0}$ and $W=10 \omega_{0}$ will be chosen to explore the geometric phase and the information flow, numerical results are shown in Fig.(1)-(6). Fig:1 and Fig.2 show the geometric phase $\Phi_{\mathrm{GP}}$ and the information flow $\mathcal{M}$ as a function of $R$ with different parameter $z$ for weak coupling $\left(W=0.1 \omega_{0}\right)$. The geometric phase $\Phi_{\mathrm{GP}}$ is plotted in units of $\pi$. We can see from Fig, 1 that the geometric phase increases monotonically with the parameter $R$. In this case, the coupling strength $W$ is small enough so that there is no information flowing back into the system in a quasi-period. For pure initial states, i.e. $z=1$, comparing Fig 1 with Fig, 2 , we find that the larger the geometric phase is, the more information flow to the environment, this is confirmed by Eq.(11). Because $\mathcal{N}(R)$ is always zero in a quasi-period, the geometric phase mainly depends on $\mathcal{M}(R)$.

With increase of the coupling strength $W$, the correlation time of the environment $\tau_{B}$ approaches to the time $T$, which indicates that $\mathcal{N}(R)>0$ in a quasi-period. In Fig 3 and Fig 4 , we plot the information flow $\mathcal{N}(R)$ and the geometric phase $\Phi_{\mathrm{GP}}$ as a function of $R$ for $W=\omega_{0}$. Here we only focus on the information flow $\mathcal{N}$, because it characterizes the non-Markovianity of the open system and describe the backward information flow. 


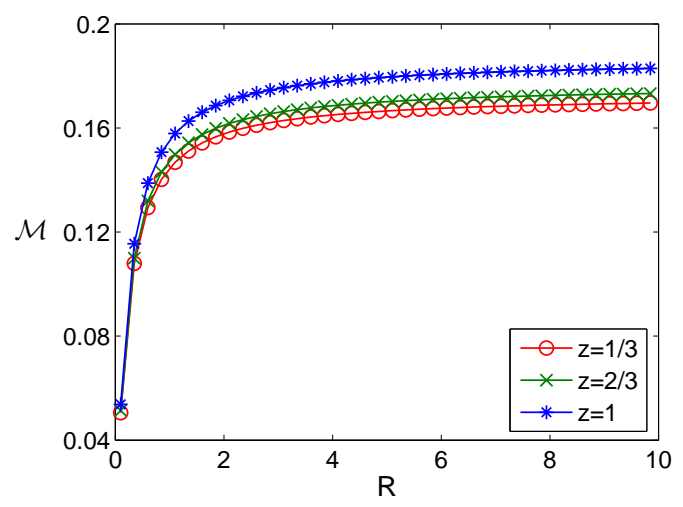

FIG. 2: (Color online) The information flow $\mathcal{M}$ as a function of the parameter $R$ with different $z$. The parameters are the same as in Fig 1

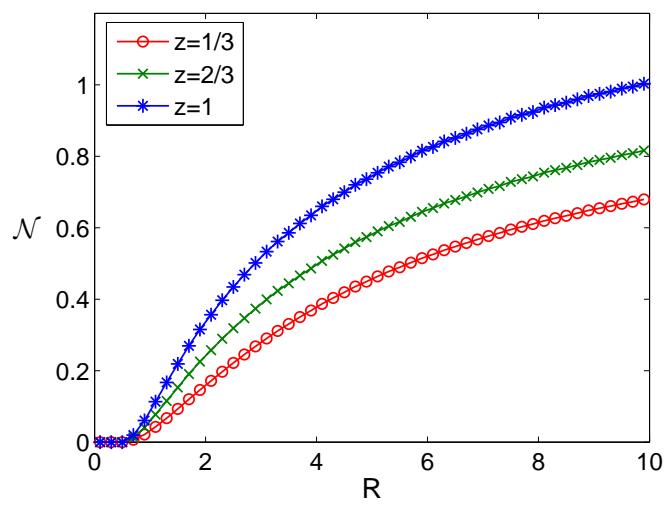

FIG. 3: (Color online) The information flow $\mathcal{N}$ as a function of the parameter $R$ with different $z$. Here the coupling strength $W=\omega_{0}$ and $\omega_{0}=1$. The initial states of the open system are chosen as Fig 1

Comparing Fig 3 with Fig 4 we may find that, when the information flows back to the system, the geometric phase decreases with the increase of $\mathcal{N}(R)$ and, in the region of $\mathcal{N}(R)=0$, the behavior of the geometric phase is similar with the case in the weak coupling limit. This indicates the backward information flow (i.e., information flow back to the open system) affects the geometric phase acquired by the open system. This phenomena can be understood as follows: For this time-local master equation, when the information flows back to the system, the Bloch vector moves toward to the north pole of the sphere, then the geometrical phase which is interpreted as the solid angle in Bloch sphere decreases.

When $W$ is very large, the system will drop into steady state after time $T$. In this case, by comparing the geometric phase $\Phi_{\mathrm{GP}}$ and the information flow $\mathcal{N}$, we can see that, when $\mathcal{N}(R)=0$ (as shown in Fig 6 ), the behavior of the geometric phase (as shown in Fig (5) is very similar to the case of $W=0.1 \omega_{0}$ and $W=\omega_{0}$ with $\mathcal{N}(R)=0$. With the increase of $R$, the information flow back into the open system and the geometric phase decreases with $R$, this is very similar to the case when $W=\omega_{0}$ in the region of $\mathcal{N}(R)>0$. Based on these observations, we conclude that for a dynamics described by the time local master equation, if the geometrical phase is inversely proportional to $R$, the dynamics must be non-Markovian. In other words, the non-Markovianity can be reflected in the geometrical phase to a certain extent. This conclusion are both valid for pure and mixed initial states.

It is seemingly that the point (in the $R$ axis) where the geometric phase arrives its extremum is exactly the point where $\mathcal{N}(T)$ begin to increase (in the following we will call it as the critical point), however, by carefully examination, we find that this is not the case. According to Eq.(11) and the definition of trace distance, we can clarify that the critical point is the very point where the integrand of the geometric phase reaches its minimum (a detail of proof can be found in the Appendix). Moreover, the integrand of the geometrical phase behaves similarly with the information flow $\mathcal{N}(t)$.

The information flow $\mathcal{N}(T)$ and the geometric phase $\Phi_{\mathrm{GP}}$ as a function of $\vartheta_{0}$ and $R$ are plotted in Fig 7 When 


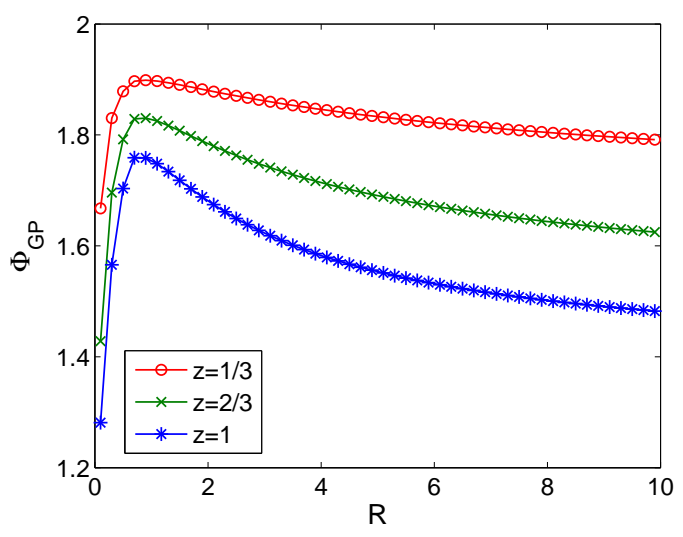

FIG. 4: (Color online) The geometric phase as a function of the parameter $R$ with different $z$. Here the coupling strength $W=\omega_{0}$ and $\omega_{0}=1$. The initial state of the open system are chosen as Fig 1

$\mathcal{N}(T)>0$, it can be seen from the figure that the more the information flows back, the smaller the geometric phase; in the region of $\mathcal{N}(T)=0$, no matter how to choose the initial azimuthal angle $\vartheta_{0}$, the geometric phase increase with $R$, which is exactly the finding of our analytical analysis. When $\vartheta_{0}=\pi / 2$, both $\mathcal{N}(T)$ and $\Phi_{\text {GP }}$ reach its extremum, in this case, the geometric phase do not change with $R$ and $\Phi_{\mathrm{GP}}=2 \pi$, but the information flow changes indeed. This can be understood as follow: when $\vartheta_{0}=\pi / 2, \sin 2 \vartheta_{0}=0$, the initial density matrix is diagonal, i.e. $\rho(0)=\operatorname{diag}\{(1-z) / 2,(1+z) / 2\}$, with this diagonal density matrix, the geometric phase is always equal to $2 \pi$ and $\mathcal{N}(T)$ changes with $R$ since the information exchange between the system and the environment varies with $R$. The situation remains unchanged for the cases where $\vartheta_{0}=0$ and $\vartheta_{0}=\pi$. Furthermore we find that, although $\Phi_{\mathrm{GP}}=2 \pi$ for both $\vartheta_{0}=\pi / 2$ and $\vartheta_{0}=\pi$, the information flows $\mathcal{N}(T)$ are completely different. This can be explained as the difference in the initial states, which are $\rho(0)=\operatorname{diag}\{(1-z) / 2,(1+z) / 2\}$ for $\vartheta_{0}=\pi / 2$ and $\rho(0)=\operatorname{diag}\{(1+z) / 2,(1-z) / 2\}$ for $\vartheta_{0}=\pi$. By the definition of the geometric phase, it depends on the spectrum of the density matrix, which are the same for the initial states, leading to the same geometric phase acquired in the dynamics. But the information flow $\mathcal{N}(T)$ for $\vartheta_{0}=\pi$ is larger than that for $\vartheta_{0}=\pi / 2$, this is because the information flow was defined as the distance between the actual state and the standard state of the open system, which are different for the initial states.

\section{B. The memory kernel master equation with exponential memory}

Now we consider the geometric phase of an open two-level system governed by the memory kernel master equation with exponential memory. Here, we just apply this model to calculate the geometric phase but do not discuss the positivity of the master equation in detail.

In the interaction picture, the integro-differential master equation with memory kernel can be expressed as

$$
\dot{\rho}(t)=\int_{0}^{t} K\left(t^{\prime}\right) \mathcal{L} \rho\left(t-t^{\prime}\right) d t^{\prime}
$$

where $\mathcal{L}$ is Liouvillian superoperator which takes the form

$$
\mathcal{L} \rho=\frac{1}{2} \gamma_{0}\left(2 \sigma_{-} \rho \sigma_{+}-\sigma_{+} \sigma_{-} \rho-\rho \sigma_{+} \sigma_{-}\right),
$$

and $\gamma_{0}$ is the dissipation rate, $K(t)$ represents the memory effect called Shabani-Lidar memory kernel[22, 23]

$$
K(t)=\gamma \exp (-\gamma t)
$$

We call $\tau_{R}=\frac{1}{\gamma}$ the memory time. It is not difficult to solve this integro-differential equation by the Laplace transform with the initial condition Eq.(16). In the Schrödinger picture, the solution is

$$
\rho(t)=\frac{1}{2}\left(\begin{array}{cc}
\left(1+r_{0} \cos \theta_{0}\right) \xi(C, t) & r_{0} \sin \theta_{0} e^{-i\left(\omega_{0} t+\phi_{0}\right)} \xi\left(\frac{C}{2}, t\right) \\
r_{0} \sin \theta_{0} e^{i\left(\omega_{0} t+\phi_{0}\right)} \xi\left(\frac{C}{2}, t\right) & 2-\left(1+r_{0} \cos \theta_{0}\right) \xi(C, t)
\end{array}\right)
$$




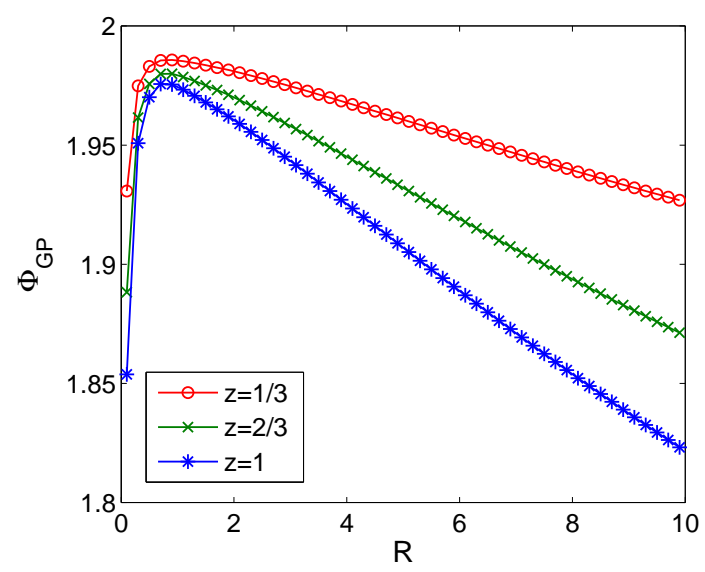

FIG. 5: (Color online) The geometric phase as a function of $R$ with different $z$. Here the coupling strength $W=10 \omega_{0}$ and $\omega_{0}=1$. The initial states of the open system are the same as in Fig 1

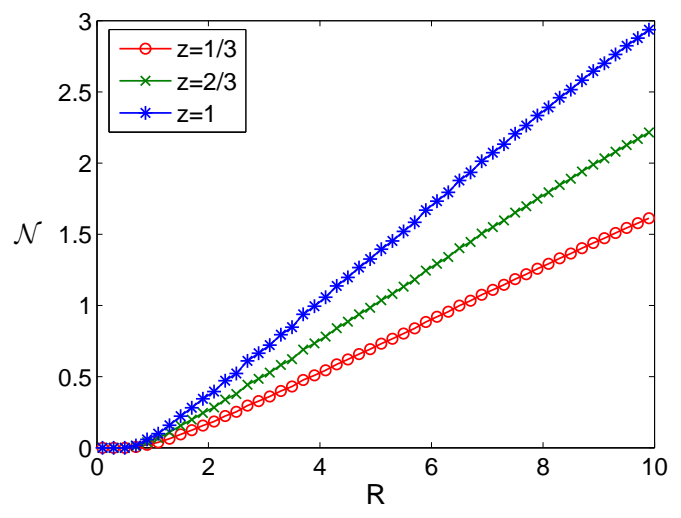

FIG. 6: (Color online) The information flow $\mathcal{N}$ as a function of $R$ with different $z$. Here the coupling strength $W=10 \omega_{0}$ and $\omega_{0}=1$. The initial states of the open system are the same as in Fig 1

where

$$
\begin{aligned}
& \xi(C, \tau)=e^{\frac{-\tau}{2}}\left[\cosh \left(\frac{\Omega \tau}{2}\right)+\frac{1}{\Omega} \sinh \left(\frac{\Omega \tau}{2}\right)\right], \\
& \Omega=\sqrt{1-4 C} .
\end{aligned}
$$

The parameters $C$ and $\tau$ are defined as $C=\frac{\gamma_{0}}{\gamma}$ and $\tau=\gamma t$.

By the same procedure as in Sec III A we establish relation between the geometric phase and the information flow, which is found to be similar to that in the last section. Hence, the conclusions for the relation between the geometric phase and the information flow hold true for open systems described by memory kernel master equation. See the numerical results shown in Fig 8 and Fig, 9 ,

For the memory kernel master equation with exponential memory kernel, it is well known that, in some region of parameter $C$, the master equation Eq.(26) may lead to non-positive density matrix. In Ref.[23], the positivity for a density matrix has been discussed: When $C>\frac{1}{4}$, the the memory kernel master equation with exponential memory is not valid, because the second perturbation used to drive the master equation does not suit this case. Recently, Breuer et al. has checked that this kind of master equation does not own memory effect when the positivity of the density matrix is conserved [24]. When $C<\frac{1}{4}$, there is no information flow back into the open system, no matter how to choose the parameter $\gamma_{0}$, so we choose $\gamma_{0}=0.1 \omega_{0}$ and plot the geometric phase and the information flow $\mathcal{M}(C)$ in this region. Comparing Fig 8 with Fig $[9$, we can find the link between the information flow $\mathcal{M}(C)$ and the geometric phase is the same as we found in Sec $\llbracket$ IIA. 


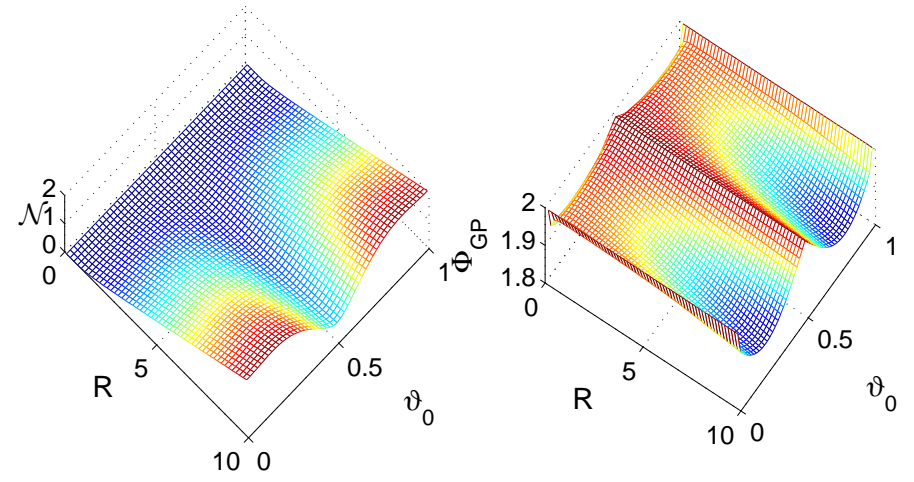

FIG. 7: (Color online) (a)The information flow $\mathcal{N}$ and (b) the geometric phase $\Phi_{\mathrm{GP}}$ as a function of $R$ and the initial polar angle $\vartheta_{0}$ (in units of $\pi$ ). Here the coupling strength is $W=10 \omega_{0}$ and $\omega_{0}=1$. The initial states of the open system are chosen as $z=1 / 2$ and $\varphi_{0}=\pi / 6$.

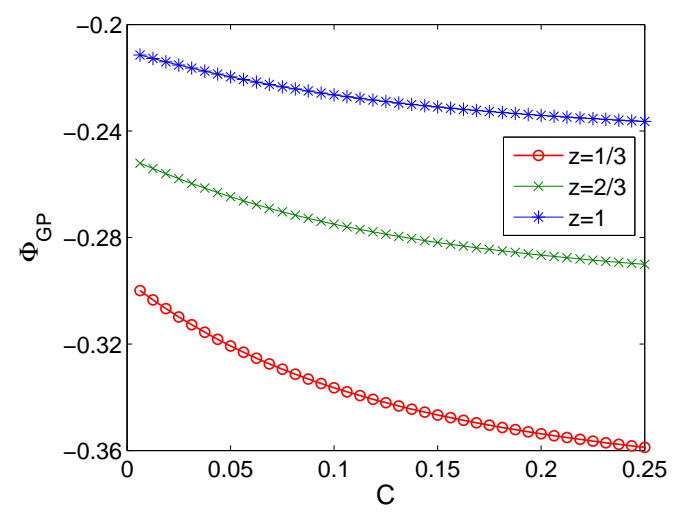

FIG. 8: (Color online) The geometric phase for the memory kernel master equation with exponential memory kernel as a function of the parameter $C$ with different $z$. Here the dissipation constant takes $\gamma_{0}=0.1 \omega_{0}$ and $\omega_{0}=1$. We choose the same initial states as in Fig 1 for the open system to plot this figure.

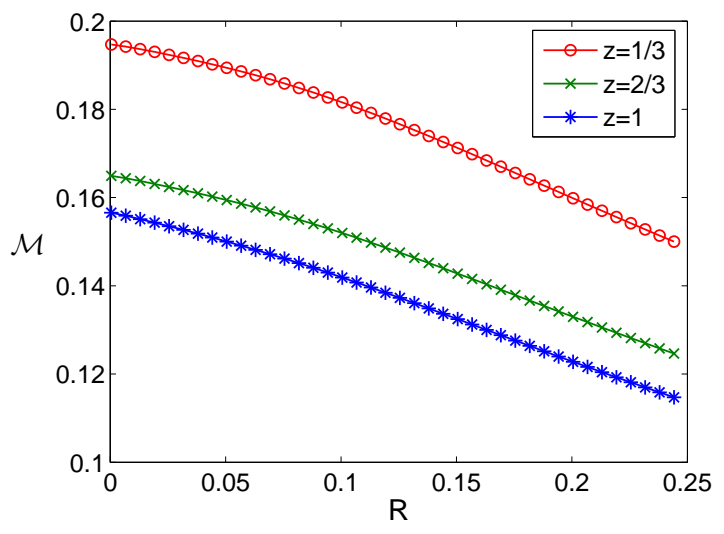

FIG. 9: (Color online) The information flow $\mathcal{M}$ for memory kernel master equation with exponential memory kernel as a function of $C$ with different $z$, where the dissipation constant is $\gamma_{0}=0.1 \omega_{0}$ and $\omega_{0}=1$. The initial states of the open system are the same as in Fig 1 


\section{CONCLUSION AND DISCUSSIONS}

In summary, we have discussed the information flows and the geometric phase in different non-Markovian process. For an open two-level system with pure initial states, an analytic relation between the information flow and the geometric pahse has been given in terms of Bloch vector. For mixed initial states, two kinds of master equation, namely time-local master equation and memory kernel master equation with exponential memory, have been numerically studied. We find that, in both cases, the information flows influence the geometric phase directly, a relation between the geometric phase and information flow is numerically established. An understanding for the observation is provided.

The forward and backward information flow are by definition different, but they complementarily describe the information exchange between the environment and the system. The backward information flow can be used to describe the Non-Markovianity of the open system, while the forward information flow was connected with the coherence loss. Neither forward information flow nor backward information flow can be measured directly, indicating that the measure of non-Markovianity defined in this way can not be directly observed in experiment. However, mixed state geometric phases are measurable and the feature caused by it has been observed 25]. This motivates the establishment of the connection between the geometric phase and the information flow. Indeed, The finding of this paper suggests that the geometric phase can reflect the non-Markovianity and then can serve as a measure of non-Markovianity for open systems.

This work is supported by NSF of China under Grant Nos. 10775023, 10935010 and 10905007.

\section{APPENDIX}

In this appendix, we show in detail that the critical point is exactly the point where $\mathcal{N}(t)$ begin to increase. By Eq.(11), we write the integrand as

$$
A(t, R)=\omega_{0}\left[\frac{1}{2}+\frac{r_{z}(t, R)}{2 \sqrt{4 D^{2}(t, R)-2 r_{z}(t, R)-1}}\right]
$$

where $r_{z}(t)=r(t) \cos \theta(t)$. To find the critical point, we take a derivative with respect to $R$

$$
\frac{\partial}{\partial R} A(t, R)=\frac{\omega_{0} r_{0}^{2} \sin ^{2} \theta_{0}}{4 r(t, R)^{3}}\left[\left(1+r_{0} \cos \theta_{0}\right)|c(t, R)|^{2}+1\right] \frac{\partial}{\partial R}|c(t, R)|^{2},
$$

noting that $r(t, R) \in[0,1]$ and $\left(1+r_{0} \cos \theta_{0}\right)|c(t, R)|^{2}+1>1$, we find, when $\frac{\partial}{\partial R}|c(t, R)|^{2}=0, A(t, R)$ must reach its extremum. Moreover, we check the first derivative of the trace distance with respect to $R$,

$$
\frac{\partial}{\partial R} D(t, R)=\frac{1}{4 D(t, R)}\left[\left(1+r_{0} \cos \theta_{0}\right)^{2}|c(t, R)|^{2}+r_{0}^{2} \sin ^{2} \theta_{0}\right] \frac{\partial}{\partial R}|c(t, R)|^{2} .
$$

Obviously, since $D(t, R) \in[0,1]$ and $\left(1+r_{0} \cos \theta_{0}\right)^{2}|c(t, R)|^{2}+r_{0}^{2} \sin ^{2} \theta_{0} \geq 0$, we can see that the trace distance arrive at its extremum if and only if $\frac{\partial}{\partial R}|c(t, R)|^{2}=0$, this is exactly the condition for the integrand to reach its maximum. Substituting Eq.(7) into Eq.(32), we obtain

$$
\frac{\partial}{\partial R} \mathcal{N}(t, R)-\frac{\partial}{\partial R} \mathcal{M}(t, R)=\frac{1}{4 D(t, R)}\left[\left(1+r_{0} \cos \theta_{0}\right)^{2}|c(t, R)|^{2}+r_{0}^{2} \sin ^{2} \theta_{0}\right] \frac{\partial}{\partial R}|c(t, R)|^{2} .
$$

At the critical point, there is no backward information flow, i.e. $\mathcal{N}(t)=0$. Thus if the critical point is the very point satisfied $\frac{\partial}{\partial R}|c(t, R)|^{2}=0$, the derivative of $\mathcal{M}(t)$ must be zero at this point. Because the property of the information flows, it is difficult to obtain an analytic results. The numerical result of $\mathcal{N}(T)$ and $\mathcal{M}(T)$ have been shown in Fig 10 . which validate our hypothesis. This is further confirmed by Fig. 11.

[1] S. Pancharatnam, Proc. Indian Acad. Sci., Sect. A 44, 247 (1956).

[2] M. V. Berry, Proc. R. Soc. London A 392, 45 (1984).

[3] B. Simon, Phys. Rev. Lett. 51, 2167 (1983).

[4] Y. Aharonov and J. Anandan, Phys. Rev. Lett. 58, 1593 (1987). 


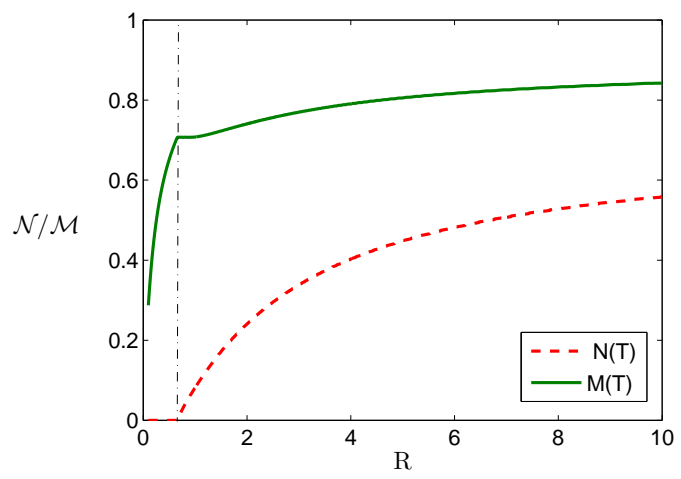

FIG. 10: (Color online) The measure $\mathcal{N}$ and $\mathcal{M}$ as a function of $R$ with a pure initial state $\left(\vartheta_{0}=\pi / 3\right)$. The coupling strength $W$ is chosen as 0.6. This figure shows that, at the start point (the point of crossover with the dot-dashed line) of the measure $\mathcal{N}(T)$, the derivative of the measure $\mathcal{M}(T)$ with respect to $R$ is zero.

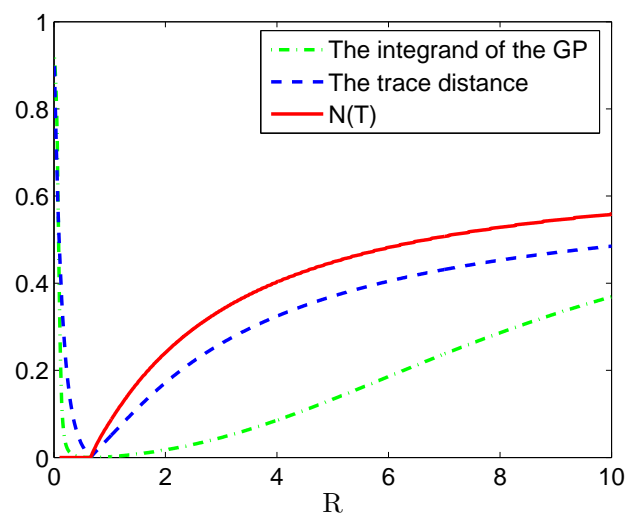

FIG. 11: (Color online) The integrand of the geometric phase $A(T, R)$, the trace distance $D(T, R)$ and the measure $N(T, R)$ are plotted in this figure. The initial state is chosen as $\vartheta_{0}=\pi / 3$ and the coupling strength $W$ is equal to 0.6 .

[5] J. Samuel and R. Bhandari, Phys. Rev. Lett. 60, 2339 (1988).

[6] N. Mukunda and R. Simon, Ann. Phys. (N.Y.) 228, 205 (1993).

[7] A. Uhlmann, Rep. Math. Phys. 24, 229 (1986); Lett. Math. Phys. 21229 (1991).

[8] E. Sjöqvist, A. K. Pati, A. Ekert, J. S. Anandan, M. Ericsson, D. K. L. Oi, and V. Vedral, Phys. Rev. Lett. 85, 2845 (2000).

[9] K. Singh, D. M. Tong, K. Basu, J. L. Chen, and J. F. Du, Phys. Rev. A 67, 032106 (2003); M. Ericsson et al, Phys. Rev. A 67, 020101(R) (2003); J. G. Peixoto de Faria et al, Europhys. Lett. 62, 782 (2003).

[10] D. M. Tong, E. Sjöqvist, L. C. Kwek, and C. H. Oh, Phys. Rev. Lett. 93, 080405 (2004).

[11] H. P. Breuer and F. Petruccione, The Theory of Open Quantum Systems (Oxford University Press, Oxford 2002).

[12] X. L. Huang and X. X. Yi, Europhys. Lett. 82, 50001 (2008).

[13] Juan-Juan Chen, Jun-Hong An, Qing-Jun Tong, Hong-Gang Luo and C. H. Oh, Phys. Rev. A. 81, 022120 (2010).

[14] H. P. Breuer, E. M. Laine and J. Piilo Phys. Rev. Lett. 103, 210401 (2009).

[15] Xiao-Ming Lu, Xiaoguang Wang, C. P. Sun, Phys. Rev. A. 82, 042103(2010).

[16] Javier Prior, Alex W. Chin, Susana F. Huelga, and Martin B. Plenio, Phys. Rev. Lett. 105. 050404 (2010).

[17] Angel Rivas, Susana F.Huelga and Martin B.Plenio, Phys. Rev. Lett. 105. 050403 (2010).

[18] E. M. Laine, J. Piilo and H. P. Breuer, Phys. Rev. A 81, 062115 (2010).

[19] V. Gorini, A. Kossakowski, and E. C. G. Sudarshan, J. Math. Phys. 17, 821 (1976).

[20] H. P. Breuer, Phys. Rev. A 70, 012106 (2004).

[21] K.-P. Marzlin, S. Ghose, and B. C. Sanders, Phys. Rev. Lett. 93, 260402 (2004).

[22] S. M. Barnett and S. Stenholm, Phys. Rev. A 64, 033808 (2001).

[23] S. Maniscalco, Phys. Rev. A 75, 062103 (2007).

[24] L. Mazzola, E. M. Laine, H. P. Breuer, S. Maniscalco and J. Piilo, Phys. Rev. A 81, 062120 (2010).

[25] J. F. Du, P. Zou, M. J. Shi, L. C. Kwek, J.-W. Pan, C. H. Oh, A. Ekert, D. K. L. Oi, M. Ericsson, Phys. Rev. Lett. 
91, 100403(2003); M. Ericsson, D. Achilles, J. T. Barreiro, D. Branning, N. A. Peters, P. G. Kwiat, Phys. Rev. Lett. 94, 050401 (2005); J. Klepp, S. Sponar, S. Filipp, M. Lettner, G. Badurek, Y. Hasegawa, Phys. Rev. Lett. 101, 150404 (2008); A. Ghosh, A. Kumar, Physics Letters A 349, 27 (2005). 